\title{
ANNA TYLUSIŃSKA-KOWALSKA
}

\author{
Università di Varsavia \\ atylusinska@uw.edu.pl
}

\section{POLACCHI IN SICILIA NEL PERIODO DEL REGIME COMUNISTA - RICORDI, 'REPORTAGES' E RELAZIONI DI VIAGGI}

\begin{abstract}
Anna Tylusińska-Kowalska, I Polacchi in Sicilia nel periodo del regime comunista ricordi, 'reportages' e relazioni di viaggi [Poles in Sicily in the period of communist regim - memories, 'reportages' and trip reports], Studia Romanica Posnaniensia, Adam Mickiewicz University Press, Poznań, vol. XLI/4: 2014, pp. 77-89. ISBN 978-83-232-2791-5. ISSN 0137-2475. DOI: 10.7169/strop 2014.414.007
\end{abstract}

Ke y w ord s: Poles in Sicily, Communist regime- memories, reportages, trip reports

The article is focused on accounts of journeys in Sicily performed by Polish columnists and historians in the period of 1949-1989. In other words it embraces almost half century of history of Poland that went through one of the most difficult moments in its days. Lack of freedom of expression, lack of freedom of movement throughout Europe resulted in a situation that only few could travel by establishing positive relationships with the communist authorities. Descriptions of Sicily from that period published by both writers (Marian Brandys, Jarosław Iwaszkiewicz) and historians (Joanna Olkiewicz), as well as publicists (Waldemar Łysiak, Marian Kałuża) present the island in a biased and shallow way. Beside Iwaszkiewicz book, which at times evokes Krasiński journals, we can deal with accounts focused on widely criticized political and economic situation. Thanks to those descriptions Polish readers, during the regime, could have consolidated their belief as to the poor life conditions in the West (particularly in Sicily). What is more there would be nothing to regret if they weren't able to reach those destinations. It constitutes a radical difference with the way of depicting Sicily in XVIII and XIX century by Polish travelers. Then the island was presented in poetic and colorful way, and the descriptions were really encouraging to travel.

Fare storia, come viaggiare, è un tipo di alienazione salutare che forma gli osservatori, intensifica la coscienza delle persistenze e delle differenze e permette a chi ritorna di rientrare in un presente prima opprimente per la sua familiarità con lo sguardo di un estraneo. Ciò che prima era il veicolo delle nostre percezioni, la lente che rifrangeva i nostri giudizi, è divenuto un oggetto, una sostanza, un mondo con il quale è forse possibile raggiungere un certo livello di integrazione, coesione e del quale si può ottenere un grado di comprensione non accessibile a chi non ha compiuto il viaggio (Leed, 1992: 35). 
Viaggio, dunque, come categoria dello spirito ed anche vicenda storica ed esistenziale. Fenomeno che si presenta particolarmente complesso. Riflettendo sul viaggio come categoria dello spirito, Mario Olivieri vede il viaggio come «intima e permanente figura dell'uomo, ontologicamente e antropologicamente considerato, e quindi ne rappresenta e somatizza le espressioni e le vicende sostanziali secondo cadenze e forme costanti, mescolate a modalità tipiche e specifiche culturali» (1992: 5). Il che significherebbe che il desiderio di spostarsi è innato all'uomo e lo accompagna per tutta la vita, generalizzando, beninteso. Accanto a chi sente dentro di sé quest'ansia di staccarsi dal proprio ambiente, staranno coloro che pur condividendo quelle modalità rimarranno sempre fissi nel loro posto, fra amore ed odio.

Nei momenti particolari della storia di una nazione il viaggio diventa anche una specie di fuga da se stessi, anzi una ricerca di se stessi attraverso il confronto con 'il diverso', oppure una conferma della propria identità. Sarà questo il caso dei Polacchi in viaggio per l'Europa nelle varie epoche storiche, anche se i motivi del cammino attraverso i Paesi nuovi saranno stati diversi. Essi si decidono quindi a ricorrere a uno sforzo, spesso esponendosi a vari rischi per confermare la propria presenza in Europa, insistendo sul fatto di sentirsi europei dal quale concetto furono esclusi per quassi mezzo secolo.

Effettivamente, esaminando il fenomeno 'viaggio' in varie epoche storiche, si notano caratteristiche ben precise. Claudio Magris da grande scrittore anch'egli, osserva:

Soltanto uscendo dalla propria casa, l'io, il soggetto, può tornarvi in modo libero e maturo; soltanto uscendo da se stesso può arrivare in modo autentico a se stesso [...] Questo viaggio è un viaggio circolare; si parte da casa, si attraversa il mondo, e si ritorna a casa, anche se a una casa molto diversa da quella lasciata, una casa che acquista significato soltanto grazie alla partenza, alla scissione originale. [...] Il viaggio diventa un modo, anzi forse il modo per eccellenza per realizzare l'antico 'conosci se stesso' (1990: 9).

Gli odepori polacchi sia nelle epoche remote che quelle moderne facevano parte della "grande famiglia" europea per dirla alla Tommaseo, che dell'Italia si fecero Mecca del loro pellegrinaggio culturale, a volte anche politico. Proprio come dice Giuseppe La Rosa nel suo libro La Sicilia come mito in Goethe e nei viaggiatori tedeschi, riferendosi alla lunga storia dell'interesse degli stranieri per l'isola:

Quella dei viaggiatori stranieri in Sicilia è una storia infinita, che inizia con Platone e ancora non si è conclusa. Ai viaggiatori antichi e moderni che, dopo avere effettuato l'esperienza della Sicilia, ne divulgano il ricordo sotto forma di lettera, diario, poesia, pittura, fotografia, la Sicilia deve la scoperta di alcune sue identità. Sembra che i viaggiatori stranieri vedano il paese in modo molto diverso da chi vi è nato e vi risiede. Lo vedono attraverso una sensibilità che rispecchia la loro cultura. È scontato che chi giunge in Sicilia da un'altra parte del mondo abbia una propria cultura. È questa cultura che gli suggerisce come deve vedere la Sicilia e quali aspetti evidenziarne. Con la conseguenza che le Sicilie sono tante quanti sono i viaggiatori stranieri in Sicilia» (1996: 5). 
La presenza polacca in Sicilia segna una tappa importante dei contatti siculopolacchi, ne restano prova le relazioni di viaggio a partire dal 1595 e soprattutto i diari di viaggio della seconda metà del Settecento che restituiscono la verità storica sull'importanza degli spostamenti degli intellettuali polacchi nelle zone più remote d'Europa, terre lontane, eppure così vicine e sentite ed è il caso, in effetti della Sicilia.

Ogni epoca dei viaggi ha le sue caratteristiche - basti pensare al Grand Tour illuministico che segnò un'assidua frequenza polacca in Sicilia, incentrato non solo sulle rovine e reperti archeologici bensì sul presente, sulla vita, sull'economia e problemi sociali. Altri saranno i viaggi dei romantici, abbiamo anche noi una relazione bellissima di Zygmunt Krasiński, viaggi contemplativi e interiorizzati, altri dell'epoca successiva: il conte Smorczewski vedrà nella Sicilia del secondo Ottocento la fonte inesauribile di ricchezze naturali non sfruttate o addirittura sprecate...

Nel periodo interbellico i turisti vi accorreranno attratti da un mucchio di curiosità storiche, archeologiche, culturali, chiuderanno invece un occhio sulla realtà fascista che imponeva comportamenti ben chiari, bloccava ogni creatività, schiacciava... Il mondo siciliano di Brancati i turisti stranieri o non lo notavano o facevano finta di non vederlo.

Di fronte alla realtà politica non del tutto neutrale o diversa da quella del paese di partenza due sono gli atteggiamenti da assumere: fare finta di non vedere e concentrarsi sulla cultura, arte, bellezza della natura o farsi coinvolgere nelle discussioni politiche optando sempre per una fazione, una ideologia, una propaganda. Inutile dire come si comportavano i Polacchi in Sicilia negli anni soprattutto Cinquanta del Novecento - non era dato loro quel primo atteggiamento 'neutrale', furono costretti a commentarla con rabbia e critica asprissima. Per gli altri, che scesero sull'Isola nei decenni posteriori si trattava invece di una scelta ideologica, dei vantaggi che comportavano certi atteggiamenti e disagio esterno se invece si andava controcorrente.

Le visite in Sicilia nel periodo direttamente dopo la guerra vengono descritte da due autori e pubblicisti di primaria importanza per la letteratura e cultura polacca: Marian Brandys (1912-1998) e, il già menzionato Jarosław Iwaszkiewicz (1894-1980).

Il primo capitò sull'Isola nel 1948 durante il suo prolungato soggiorno in Italia in qualità di corrispondente della statale Agenzia di Stampa e Informazione [Agencja Prasowo-Informacyjna], il frutto di quel soggiorno fu il volumetto di tipo reportage intitolato Spotkania włoskie [Incontri italiani]. Il libro ha una storia editoriale abbastanza curiosa: pubblicato per la prima volta nel 1949 dalla casa editrice 'Czytelnik' ; invece nel 1953, l'anno della morte di Stalin escono quasi parallelamente due 'seconde edizioni': in marzo quella per, come dice l'autore stesso 'i giovani', lo pubblica la casa editrice specializzata in editoria per adolescenti

\footnotetext{
${ }^{1}$ Sotto il regime comunista tutte le case editrici erano statali.
} 
e gioventù 'Nasza księgarnia', invece in agosto lo pubblica nuovamente 'Czytelnik'. Sarebbe molto interessante paragonare dettagliatamente le due versioni che risulta impossibile in questa sede per mancanza di spazio: comunque quella dedicata alla gioventù polacca che con entusiasmo deve guardare alla nuova realtà è corredata con note cioè spiegazioni e soprattutto con la Premessa dell'Autore il quale, con la retorica filocomunista oltrepassa tutti i limiti, oltrepassa se stesso.

Certo, Brandys è giovane anche lui, ma non giovanissimo, bada alla sua carriera, ma forse sarebbe il caso di aprire la parentesi della sua biografia: proveniente da una famiglia di 'intellighenzia' di origine ebraica, durante la guerra impegnato militarmente nelle file di un gruppo operativo ideologicamente neutrale, salvatosi dal campo tedesco di oflag visse e pubblicò le sue opere a Varsavia. Subito dopo la guerra, uno dei primi membri del partito comunista fra della sua generazione e del suo gruppo professionale iniziò la carriera giornalistica, divenne portavoce della propaganda del regime. Scrive nella Premessa e quello che salta subito agli occhi non è solo la retorica, bensì il tono e l'entusiasmo da cui sembra essere sinceramente trasportato. Inizia nel modo seguente la sua 'chiacchierata' con i giovani:

Nel 1949 sono partito per l'Italia come corrispondente speciale di una delle agenzie di stampa polacche. Vi ho soggiornato per 5 mesi dopodiché la polizia del ministro Scelba colpita fino in fondo dal contenuto dei miei articoli pubblicati sui giornali polacchi mi ha consegnato, senza una minima giustificazione l'ordine dell'immediato ritorno in Polonia. Il soggiorno di 5 mesi in qualità di reporter, viaggi in lungo e in largo sulla Penisola Appenninica è stato per me la lezione di storia dell'Italia contemporanea - storia di una nazione venduta dal suo governo agli imperialisti americani, storia di una nazione che combatte eroicamente per la libertà, per il lavoro, per il pane. [...]. Imparavo la storia dell'Italia contemporanea osservando piazze e strade italiane durante i giorni delle lotte accanite contro il Patto Atlantico. Sandro R. e altri artisti italiani di idee progressiste di via Margutta mi introducevano nella tragedia della cultura italiana che perisce sepolta sotto il mucchio di prodotti americani senza alcun valore. [...]. Marco G., distributore del giornale «Unità» e Antonia P., operaia della fabbrica di sigari mi raccontavano della lotta del proletariato italiano (1953a: 5-6).

Brandys si decide per un discorso rapido, immediato, coinvolgente, ammiccante con il giovane lettore. Intende di essere apprezzato per il suo approccio, tendenzioso che fosse, alla realtà italiana di allora. Quel che risulterà caratteristico e riguarderà la maggior parte dei relatori polacchi dei loro soggiorni siciliani sarà impegno politico, appunto. Li vedremo aggiornati sui nomi, cognomi, ruoli ricoperti. Con leggerezza e brio letterario tipico per lo scrittore viene raccontata 'la sua' Italia, tale da essere ricordata da egli stesso, tale da essere trasmessa ai giovani:

Umida del sangue operaio la terra dell'Emilia, le campagne siciliane che gemono in preda assoluta della mafia, gli operai di Torino e di Taranto che si difendono nelle loro fabbriche come nelle fortezze, i senza tetto che vivono nelle grotte di Monte Caprino di Roma e i lazzaroni di Napoli che muoiono di fame, gli scioperi degli operai di fabbriche e degli operatori di campagne che non finiscono mai, il rombo degli scooter 'celere' che fanno stragi 
tra le folle che manifestano per vie e piazze in difesa dei loro diritti costituzionali - tutto questo mi disegnò l'immagine della povertà e decadenza del governo De Gasperi e Scelba e della grandezza ed eroismo della nuova Italia capeggiata da Palmiro Togliatti e Pietro Nenni.

La Premessa continua poi con una visione sempre più cupa dell'Italia, l'Autore sottolinea che passati tre anni, la situazione del paese è peggiorata e va dritto verso la distruzione e si avvicina all'abisso. Con quell'impegno e fedeltà al regime è difficile poi credere che Brandys Marian, magari seguendo la scia del fratello più giovane Kazimierz (nato nel 1916) anch'egli con alle spalle una breve parentesi del partito comunista, il quale tuttavia ben presto passò all'opposizione di cui già negli anni Sessanta divenne icona, anche Marian, alla fine degli anni Settanta si avvicina all'opposizione anticomunista ${ }^{2}$ e verrà visto, negli anni a seguire, come uno degli uomini di Solidarność.

Il testo sopraccitato conferma soltanto le contraddizioni e la strada difficile degli intellettuali polacchi verso le posizioni ben chiare, e tanti furono che dagli apologeti del regime passarono in seguito all'opposizione quella più militante ed accanita che dovette costare loro anni di carcere. Bisogna tuttavia riconoscere che non tutti gli intellettuali alle soglie della carriera professionale diventavano così ligi al regime come appena citato Marian Brandys. Gli incontri italiani sono dunque una relazione propagandistica e soggettiva dell'Italia del 1949 , un commento filocomunista al 'capitalismo marcio e squallido', e solo poche volte, descrivendo le sue passeggiate romane l'autore si lascia trasportare dalle emozioni estetiche, dal fascino del posto e ne parla con piena sincerità. Ma sono poche righe, il clima che domina le descrizioni della Città Eterna è quello dei Ragazzi di vita di Pasolini, romanzo pubblicato sei anni più tardi quindi non poteva esserne modello.

La Sicilia invece è terra di delinquenza e della mafia. Brandys le dedica l'ultimo capitolo del libro, 13 pagine che bastano però per raccontare tutto il male che corrode l'Isola. Il lettore dopo averle lette saprà benissimo di non voler mai mettervi piede, correndo rischi gravissimi di esser ucciso dalla mafia in pieno giorno, per strada, con il tacito consenso di chi vi passa accanto che forse consenso non è, ma è paura. Perché in Sicilia tutti hanno paura di tutto, vivono in terrore, non solo in povertà. Il capitolo inizia con una scena teatrale quasi in cui il cicerone si ferma, si blocca e all'improvviso finisce il lavoro spiegando al gruppo di turisti che dopo l'imbrunire c'è il pericolo di banditi. E lo conferma l'accompagnatore dello scrittore, citato di nome, dr Fabio Ardi, eppure si trovano a Palermo e sono le cinque di sera. L'Autore non si dimentica di osservare che gli sembra questo un comportamento strano sicché la guida è sicuramente avida di soldi, come tutti i siciliani. Brandys informa poi scientificamente il pubblico polacco sulla storia della mafia e sullo stato

\footnotetext{
${ }^{2}$ Si tratta di KOR (Comitato per il Soccorso degli Operai) fondato nel 1976 dagli esponenti dell'opposizione anticomunista.
} 
attuale di cose. Ne descrive strutture, attività, settori di operazione. Per illustrare meglio il problema passa subito al caso reale:

Durante la mia permanenza in Sicilia successe un incidente a Partinico: 'Capo' (il comandante della mafia locale - spiega tra parentesi) mirando a punire un uomo che per sfortuna sua si era trovato tra i piedi della mafia in qualità di nemico, gli mandò dietro tre banditi promettendo loro un lauto guadagno e una totale impunità in caso riuscissero a compiere un lavoro 'pulito'. Ma, dopo che il lavoro fu eseguito anziché pagarli come stava nei patti li espose all'interrogatorio in commissariato, avendoli denunciati. Purtroppo aveva anche sottovalutato il fenomeno di omertà perché tre giorni dopo i tre banditi, rispettando tute le procedure, lo trasportarono via da casa e lo impiccarono nella piazza centrale di Partinico (1953b: 131).

Una parte del racconto 'siciliano' viene dedicata a Salvatore Giuliano il cui mito aveva conquistato tutt'Europa. Della Sicilia il lettore polacco quindi non imparava nulla: Brandys così chiude la parte 'siciliana' del suo racconto sull'Italia:

Uno degli eminenti rappresentanti del Partito Popolare siciliano mi disse il giorno della mia partenza: [...] L'unico modo per sradicare il male è la realizzazione di una largamente intesa riforma agraria. Pochi anni dopo l'esecuzione della riforma non ci sarà più in Sicilia né mafia né delinquenza organizzata, né banditismo. Priva della sostanza indispensabile per la sua esistenza morirà con una morte naturale (1953b: 134).

La storia dimostrò che non era del tutto vero...

La relazione di Brandys, talmente propagandistica e tendenziosa costituisce tuttavia per un tono della narrazione che, ribadiamolo, non fu imposto dal regime, bensì scelto apposta dal pubblicista, un'eccezione tra altre descrizioni dei soggiorni dei Polacchi sull'Isola prima del 1989.

Chi viaggia negli anni Quaranta, Cinquanta, Sessanta in Polonia, sono i pochi eletti, ma questi pochi eletti, si sentono in dovere di condividere le proprie osservazioni e impressioni con i lettori per i quali il testo scritto rimane il più delle volte l'unica fonte di conoscenza della Sicilia. Ma il libro sulla Sicilia sarebbe incompleto se si cercasse di evitare di presentarne i problemi, difficoltà economiche e infine la mafia, la malavita che resta per chi in qualche maniera collabora al regime un punto fermo di appoggio, una ragione per cui andarci. Il caso classico di un testo che in questo modo descrive l'Isola è Książka o Sycylii [Il libro sulla Sicilia] di uno dei maggiori scrittori e poeti polacchi, Jarosław Iwaszkiewicz ${ }^{3}$. Iwaszkiewicz, innamorato dell'Italia e della Sicilia in particolare, dipinge un quadro bellissimo di quel posto così denso di spunti per la scrittura, per la riflessione sul passato, sull'oggi. E dentro quel libro poetico-filosofico, l'ultimo capitolo inaspettatamente si concentra su ciò che è brutto, sconvolgente, inaccettabile di quella Sicilia tanto amata,

${ }^{3}$ Il libro è uscito intraduzione italiana nel 2013, pubblicato da Messagea (Messina) trad. Francesco Groggia, prefazione di Jarosław Iwaszkiewicz. 
altrimenti posto di paradiso. Il libro sulla Sicilia ha come editio princeps l'anno 1956 ed è quindi palese sottolineare il fatto che altrimenti non sarebbe mai stato pubblicato anche se Iwaszkiewicz stesso si è piegato di fronte al regime, accettando le sue regole, partecipando attivamente alla propagandistica vita culturale di quel tempo. Si avverte tuttavia la forzatura di quel capitolo che chiude il libro, la stanchezza psicologica dello scrittore che avrebbe preferito continuare le sue riflessioni sul popolo siciliano anziché puntare un dito su quello che è margine della vita, non la sua essenza. Eppure, ligio com'era la regime, accetta le sue regole, china il capo e scrive quel che oggi probabilmente non avrebbe mai scritto o se ne avesse scritto qualcosa, il tono sarebbe stato ben diverso. Ma nel testo di Iwaszkiewicz si avverte una nota di ottimismo dettata magari dall'amore suo per la Sicilia:

Le forze minacciose si nascondono dunque nel seno di questa terra meravigliosa, terra di tragici contrasti. Ma il popolo siciliano sa lottare per il proprio futuro. Bisogna credere che questo futuro, nonostante la mafia ed ogni tipo di banditismo, nonostante il petrolio il quale, come sostiene il poeta americano Lopez Velarde 'fu inventata dal diavolo', nonostante tutto che impedisce a quest'isola di vivere felice - questo futuro porterà la mia adorata Isola sulla via piena di luce e bellissima (Iwaszkiewicz, 1956: 203).

In chiusura, tuttavia, Iwaszkiewicz torna se stesso: poeta, malinconico, sensibile alle bellezze della natura siciliana, grande amante della letteratura siciliana (cita Verga, Pirandello, Vittorini) e conclude osservando che spera che il lettore ne tragga qualcosa per sé, si arricchisca interiormente leggendo quelle righe come lo scrittore stesso visse profondamente ogni sua permanenza siciliana.

Negli anni Sessanta l'unica relazione della vita (o malavita) siciliana resta la traduzione di Il giorno della civetta di Leonardo Sciascia, mentre negli anni Settanta, si parlava non poco della Sicilia. I libri usciti in quell'epoca erano relazioni di viaggi scritte dai pubblicisti che volevano, ancora una volta affermarsi sul mercato e, soprattutto, volevano piacere ai datori di lavoro, cioè alle autorità comuniste. L'ottica quindi è più che esplicita, il tono di condanna e critica, la gioia di essere venuti da una realtà diversa, amica del popolo, amica dei poveri, sensibile alla miseria, paese in via di sviluppo che, beninteso, in Sicilia non si avvertiva, al contrario ${ }^{4}$.

Tra i relatori non mancano le donne e sono due storiche, con una carriera accademica alle spalle, con ormai diverse pubblicazioni specialistiche. Venire in

\footnotetext{
${ }^{4}$ Dopo i libri di Brandys e Iwaszkiewicz uscirono altri racconti del soggiorno in Sicilia di: W. Łysiak, Wyspy zaczarowane [Isole incantate], Instytut Wydawnicz 'Pax', Warszawa 1974; D. Dolci, Sycylia od środka [La Sicilia dal di dentro], [vers. originale: Banditi a Partinico, Inchiesta a Palermo, Spreco, Chi gioca solo], Iskry, Warszawa 1974; R. Kałuża, Zejście $z$ autostrady stońca [L'uscita dall'Autostrada del Sole], Krajowa Agencja Wydawnicza, 1981 (è il resoconto di un lungo soggiorno siciliano - 6 mesi svoltosi nel 1979).
} 
Sicilia da donna significava un bel coraggio, un contrapporsi alla propaganda ufficiale, agli stereotipi che, sotto l'influsso di questa propaganda immedesimavano la Sicilia solo con la malavita e la mafia. Fu il decidersi, l'intraprendere un tale viaggio che distingueva all'epoca le donne amanti di cultura, dell'arte, affascinate dall'antichità. Significava poi una curiosità infinita per scoprire il 'diverso', l'altra cultura, l'altra realtà, capirla, per affrontare magari con più serenità la realtà propria.

Nel dopoguerra polacco le soluzioni per fornire un testo di viaggio di qualsiasi paese occidentale si presentavano due: scrivere testi scientifici per un gruppo ristretto di lettori o conquistarsi un pubblico più vasto a costo di piegarsi alle regole del gioco imposte dal regime. La prima soluzione la sceglie una studiosa, storica e archeologa, Ludwika Press, che nel 1978 pubblica un libro intitolato Pożegnanie $z$ Minosem [Un saluto al Minosse] che ha un sottotitolo: Dal diario di viaggio nell'antico passato della Sicilia. L'autrice mantiene perfettamente la parola: percorre il suo iter attraverso l'Isola seguendo le tracce dei popoli antichi, ammirando i residui del loro passaggio, templi, teatri antichi, veniamo così a conoscere la complessa storia della Sicilia a partire dall'antichità, la sua cultura, ci sentiamo successori di quei popoli senza il cui contributo intellettuale ed artistico nonché il sapere politico ed economico l'Europa di oggi avrebbe una fisionomia del ben diversa. Descrivendo così l'Isola, l'autrice resta radicata nell'antichità, nel passato, immersa in quel patrimonio intellettuale che la affascina, ignorando o fingendo di ignorarlo, completamente il presente. Possiamo quindi desumere che non avesse grossi problemi con la censura. D'altronde leggendo il libro si ha impressione che la Sicilia non abbia semplicemente nessun presente.

Prendiamo ugualmente le Wyspy zaczarowane [Isole incantate] (1974) di Waldemar Łysiak, noto scrittore e autore di monografie storiche romanzate, architetto di professione. Il suo libro sull'Italia è concentrato più che altro sull'aspetto urbanistico e architettonico quindi ideato sulla stessa scia. Ma il fascino della Sicilia lo porta anche sull'isola e il fascino misterioso della mafia lo porta a dare il titolo ad uno dei capitoli del suo libro «Mafioso». L'incuriosito lettore scopre subito che si tratta dell'incontro dell'autore, in Sicilia, beninteso, con un archeologo che lavora 'in proprio', cioè organizza gli scavi impiegando operai, e rivendendo ai musei i pezzi antichi, presentandosi come collezionista privato. Łysiak sconvolto all'inizio da quella procedura, dopo qualche giorno trascorso in Sicilia dove, dice, tante cose impossibili sono possibili, alla fine del capitolo cambia completamente idea e il professore archeologo gli appare addirittura simpatico. La Sicilia in quegli anni andava sempre più di moda in Polonia. Vi contribuì non poco la pubblicazione del libro di Danilo Dolci cioè la raccolta di testi: Banditi a Partinico, Inchiesta a Palermo, Spreco, Chi gioca solo sotto un titolo comune: Sycylia od środka [La Sicilia vista dal di dentro]. 
Sotto una veste invece fortemente propagandistica si presenta un libro pubblicato nel 1975 scritto da una studiosa della storia d'Italia, Joanna Olkiewicz, intitolato Sycylia $w$ kalejdoskopie historii i legendy [La Sicilia nel caleidoscopio della storia e della leggenda]. Qui, come nel caso di Iwaszkiewicz e della Press abbiamo a che fare con un libro dedicato esclusivamente alla Sicilia. Nell'introduzione già leggiamo che la Sicilia è un caleidoscopio della storia - dopo aver elencato tutte le dominazioni straniere, a partire dai Fenici e dai Greci, L'Autrice, prima di chiudere il suo cammino attraverso la cultura e i monumenti siciliani si sofferma per un attimo sul presente e il presente è la mafia, beninteso.

Chissà se la censura avrebbe autorizzato la pubblicazione di un testo concentrato unicamente sulla cultura e sull'arte, senza cenno alla complessa situazione attuale. E quindi in Sicilia regna la mafia e sorvolare sull'argomento avrebbe significato che la pubblicista non capì nulla della terra che aveva percorso e descritto. Le riflessioni sull'argomento iniziano a pag. 260 e continuano per una decina di pagine per concludersi con la constatazione ambigua piuttosto che per un Siciliano la famiglia conta in prima luogo. Viene spiegato per bene il fenomeno, tipico per l'Isola, raccontata la storia di un medico che uccide un ragazzo, testimone di un omicidio mafioso. Si passa successivamente al concetto di 'omertà', per passare poi alle divagazioni sulle frodi elettorali. La Democrazia Cristiana appare come il partito che in qualche modo sostiene e dà una mano (non sempre pulita) ad alcuni politici. Ma si intravede ormai una luce nel tunnel. La commissione antimafia che opera dal 1962 registra i primi successi, molti dei boss della mafia sono già stati arrestati, tra cui Genco Russo di Mussolmeni, cito dopo l'Autrice. Un po' di spazio viene anche dedicato alla scomparsa del giornalista Di Mauro che indagava sulla morte di Enrico Maffei, in conflitto con la mafia per aver promosso la convenzione con l'Unione Sovietica per il fornimento del petrolio. La storica sembra quindi ben informata, o almeno così appare, su cose siciliane.

La politica, come si può ben constatare, alla fine è presente. Si parla in seguito dell'emigrazione, dell'esodo di massa dalla Sicilia nel 1960 verso il nord d'Italia, citati come esempi 'classici' vengono Brancati e Vittorini che ben sappiamo non sono informazioni precise. Lo stesso modo di abbandonare l'Isola, terra di origine, viene attribuito a Salvatore Quasimodo, accennando al suo iter professionale e il lavoro a Milano, senza dimenticare di sottolineare il rango del poeta e il suo Nobel nel 1959. Dopodiché leggiamo:

Ai pochi scrittori che rimasero sull'isola appartiene 'il vecchio Gattopardo', Tommasi di Lampedusa considerato poi un uomo strano. Anche se la maggior parte degli intellettuali e scrittori lasciarono l'isola, questa 'misteriosa, senza pietà, pronta per la vendetta e bellissima' (L. Sciascia, Il giorno della civetta) dà un singolare contributo all'Italia contemporanea, un elemento attorno al quale nacque e dura sempre la leggenda trasmessa soprattutto dalla letteratura. 'La Sicilia è incredibile' - dice il giovane poliziotto che viene 
dall'Italia del Nord, responsabile dell'indagine riguardante l'omicidio in un paese di Sicilia, paese dove regna la mafia - il protagonista dell'appena citato romanzo di Sciascia il quale è nato in Sicilia e le è rimasto fedele (Olkiewicz, 1975: 264).

Da questo brano possiamo ben desumere che gli intellettuali polacchi leggessero i romanzi di Sciascia per poi servirsene anche propagandisticamente. La Olkiewicz poi, con sempre ben tendenzioso punto di vista, rispettando l'occhio vigile della censura, passa in rassegna altri autori siciliani le cui opere conobbe sicuramente grazie alle traduzioni polacche. Menzionando a pagina 265 la Sicilia di Vitaliano Brancati (riporta alcuni brani da Don Giovanni in Sicilia, un altro romanzo 'siciliano', tradotto in polacco) ne sottolinea l'aspetto cupo, ma al contempo tra mistico e fantastico. Le riflessioni sulla situazione politica in Sicilia si concludono con l'osservazione sull'inefficacia della riforma agraria, sulla miseria e sulla fame che costituiscono il dì presente dell'Isola. Non manca poi l'elogio dell'opera sociale e pubblicistica di Danilo Dolci e così il circolo informativo sembra proprio chiudersi sull'argomento della mafia. Il libro della Olkiewicz mirava quindi a dare uno sguardo assai vasto sulla realtà siciliana, da apprezzare, anche se non peccano di precisione, risultano le informazioni culturali e letterarie.

Nel 1979 appare il libro sull'Italia di Roman Kałuża, Zejście z autostrady stońca [L'uscita dall'autostrada del Sole], relazione del lungo (circa di un anno) soggiorno in Italia e quindi il paese visto dal di dentro, situazione che sin dall'inizio sembra fuori dal comune. L'Autore, sotto il regime comunista, con un visto turistico di un mese (lo confessa serenamente), rimane sulla Penisola ed anche sulle isole circa un anno, lavora in vari posti svolgendo mestieri più strani. Tutto questo nel 1978, quando per uscire dalla Polonia si facevano ancora salti mortali e percorsi burocratici chilometrici. Poi, tornato in Patria, pubblica la sua relazione di viaggio in forma di 'reportage-cronaca di fatti', come se nulla fosse successo, come se fosse stato assente per un mese, come prevedeva il visto. Alla generazione che ha vissuto gli eventi di 'Solidarność' appare lievemente sospetta questa facilità di 'essere perdonato' per aver infranto le norme imposte rigidamente dal regime 5 .

Il racconto di Kałuża inizia nel 1978, l'autore giunge in Italia proprio nel tragico momento storico: Aldo Moro, rapito dalle Brigate Rosse rimane imprigionato, poi ucciso. Il 'reporter' polacco, chiamiamolo in questo modo, riporta dunque gli echi dalla stampa, sembra disporre di una relativa padronanza della lingua (le parole italiane vengono spesso inserite nel testo anche se a volte con degli errori basilari). Cerca lavoro, poi viene assunto dal proprietario di una piccola società di trasporti di Catania responsabile di trasporto merci, articolo prodotti a Verona pertanto come camionista percorre regolarmente la tratta Catania-Verona. Il libro sull'Italia di forte (2014).

${ }^{5}$ A questo proposito cfr. la monografia di Vincenzo Grienti, Operazione 'Solidarnosc' 
stampo politico, l'autore acculturato ${ }^{6}$, al corrente di novità letterarie, come libri di Sciascia, è volto soprattutto al presente. E quindi abbiamo due mondi a confronto: lo squallido e crudele capitalismo che blocca l'uomo nella sua creatività e felicità e quello comunista positivo incentrato sull'uomo, sistema politico sensibile alla povertà. Diamone un esempio: «Attraversiamo l'Emilia, passiamo dalla rossa Bologna il cui sindaco comunista ne fece un modello di ottima amministrazione, bellezza urbana e ordine, ideale assistenza sociale ed anche innumerevoli agevolazioni per i cittadini (la comunicazione urbana gratuita)» (Kałuża, 1979: 104). Stranamente il frammento rimanda al testo riportato all'inizio di Marian Brandys, eppure sono ormai passati 25 anni, una generazione intera. Nel momento in cui Kałuża presenta il suo 'reportage' Brandys organizza con altri dissidenti le strutture del KOR...

La Sicilia agli occhi di Kałuża appare, ed era da aspettarselo, come una terra gestita male, povera, con i delinquenti (mafiosi, beninteso) che spadroneggiano, dove l'omertà blocca qualsiasi tentativo di procedere sulla via legale, perché i testimoni tengono sempre la bocca chiusa. Diamo parola all'Autore:

Quando in un piccolo paese nei pressi di Palermo fu ucciso un contadino in una sparatoria, nel pieno giorno e parenti ed amici che assistevano all'assassinio scomparvero in un batter di ciglio. Non era per paura, era per prudenza. [...] Un sentimento atavico impone ai siciliani, per carattere vivaci e violenti di infilarsi, nei momenti di pericolo, una maschera di indifferenza, di noia, li priva di curiosità, di coraggio, di volontà di vivere. Lo conferma in qualche modo l'esempio di uno dei più grandi scrittori italiani - di Leonardo Sciascia nato a Racalmuto in Sicilia che ci vive finora. Nel momento del rapimento di Moro, Sciascia ha mantenuto l'indifferenza e la neutralità, similmente poi ad Alberto Moravia, ma aggredito da varie parti ha confessato allargando le braccia: «siamo condannati alle continue bugie, anche quando si tratta di omicidi dei privati e quindi anche degli statisti. [...]» (1979: 73-74).

Come si vede, Kałuża dà un'immagine storta della situazione, anche del clima politico italiano del momento e forse soprattutto, di Leonardo Sciascia, che fu proprio uno di quelli che si pronunciarono ad alta voce in merito, anzi reagì subito $\mathrm{e}$ in modo ben concreto e trasparente.. Ma Kałuża conosce lo scrittore, più avanti nel suo libro parlando ancora di mafia cita Todo modo e ricorda che ce ne era stata eseguita la traduzione polacca... Ma siccome i problemi della Sicilia contemporanea occupano almeno la metà del libro, il pubblicista di Varsavia, alla pagina 119, torna nuovamente a Leonardo Sciascia e fa cenno alla sua produzione letteraria: «Chi ha letto Todo modo di Leonardo Sciascia può già avere un'idea sull'argomento [l'argomento sarebbero i legami mafiosi tra la mafia e il potere]. Dirò soltanto che l'omicidio descritto nel libro di Sciascia non è il più caratteristico per quel genere di dispute teologiche e meditazioni che succedono nella vita reale. $\mathrm{E}$ in che cosa invece consistono?» (1980: 117-118).

\footnotetext{
${ }^{6} \mathrm{Nel}$ testo compaiono anche riferimenti alla storia antica, monumenti, ecc.
} 
Come risulta da queste poche citazioni Kałuża appoggiava esplicitamente il regime, stava da parte di chi lottava con 'Solidarność', rappresentando la Sicilia come regno della mafia e quindi della delinquenza e della corruzione. Su questo sfondo, a parer suo, operavano i deboli, come Leonardo Sciascia, appunto, che con la loro voce non erano in grado di alzarsi al di sopra di questa massa dei corrotti. Sulla Penisola, poi, la situazione non era molto migliore, irrisolto il problema delle Brigate Rosse, la debolezza dello Stato italiano. Così il lettore polacco di questo testo che fu disorientato e non capiva più nulla della situazione siciliana.

In un tono ben diverso si pronuncia sulla Sicilia e sui grandi siciliani Józef Dużyk, storico, scrittore e pubblicista, che lavorò in qualità del Direttore all'Accademia Polacca di Roma per ben 8 anni, dal 1974 al 1982. Sono del 1985 le sue Wędrówki włoskie [Passeggiate italiane]. Nel capitolo dedicato alla Sicilia si concentra sugli aspetti artistici dell'Isola e sui personaggi importanti del mondo della cultura che ne provengono. Tra gli scrittori, forse meglio conosce Tommasi di Lampedusa cui dedica sette pagine, ma anche gli altri gli sono noti, dice:

Scrivendo di Lampedusa non si può dimenticare che proprio la sua terra natale diede al mondo sia i primi esempi della poesia lirica in Italia, nel periodo tra quarto e il settimo decennio del XIII secolo ed anche dopo, su quest'isola crebbero i grandi scrittori dell'Italia: Giovanni Verga, Luigi Pirandello, Luigi Capuana fino ai nostri contemporanei: Elio Vittorini, Salvatore Quasimodo, Leonardo Sciascia» (1985: 116).

Nell'85 non si avvertiva più il bisogno di ingrandire i problemi italiani, tanto i Polacchi ormai viaggiavano parecchio e della realtà in cui si imbattevano potevano farsi le idee da soli. Quello che conta in alcune relazioni degli anni '70 e ugualmente in quell'ultima era anche l'approccio alla letteratura siciliana, nonostante le opinioni di Kałuża su Sciascia più che critiche.

Riassumendo: la Sicilia era utile alla propaganda comunista, come anche utile si rivelò Sciascia appunto con i suoi romanzi, tanto generosamente introdotti sul mercato del libro in Polonia. Le descrizioni dell'Isola si presentano estremamente diverse rispetto a quelle delle epoche precedenti. Nessuno dei 'viaggiatori' qui riportati, tranne magari Iwaszkiewicz, vede la bellezza del posto, il suo fascino, la poeticità. Li vedevano invece $\mathrm{i}$ viaggiatori sette e ottocenteschi. L'immagine della Sicilia è piatta, anche nel senso lato, visto che manca qualsiasi cenno alle montagne, all'Etna, un'immagine scolorita, anzi, grigia. La Sicilia con infiniti problemi economici, contraddizioni, storia complicata, abbandonata alla propria sorte dai governi capitalisti del dopoguerra, la politica della Democrazia Cristiana, la povertà, la malavita, la corruzione andava benissimo. I Polacchi, avendo letto di come funziona questa regione, anche se bellissima e interessante, ma sottosviluppata e misera, dovevano apprezzare di più quel che il comunismo stava facendo per loro 
e per la loro felicità. E così passava loro anche qualsiasi voglia di viaggiare in Occidente, il sogno proibito, per i più mai realizzato: la Sicilia incarnava un diamante nascosto e perso nel fango e dal fango nessuno l'avrebbe mai tratta fuori.

\section{BIBLIOGRAFIA}

BRANDys, Marian (1953a): Spotkania wtoskie [Incontri italiani]. Warszawa: Wyd. Nasza Księgarnia.

BRANDYS, Marian (1953b): Spotkania włoskie [Incontri italiani]. Warszawa: Czytelnik.

BRILLI, Attilio (2006): Il viaggio in Italia. Bologna: Il Mulino.

Di MATteo, Salvo (2000): Il grande viaggio in Sicilia. Viaggiatori stranier nell'Isola dagli Arabi ai nostri giorni, Introduzione Orazio Cancila. Palermo: Ed. Arbor.

DolCI, Danilo (1974): Sycylia od środka, trad. A. Konicki. Warszawa: Iskry.

DuŻYK, Józef (1985): Wędrówki włoskie [Passeggiate italiane]. Warszawa: PAX.

GRIGENTI, Vincenzo (2014): Operazione 'Solidarnosc'. Caltanissetta: Salvatore Sciascia Editore.

IwASZKIEWICZ, Jarosław (1956): Książka o Sycylii [Il libro sulla Sicilia]. Kraków: Wyd. Literackie.

KAŁUŻA, Roman (1979): Zejście z autostrady słońca [L'uscita dall'autostrada del Sole]. Warszawa: Krajowa Agencja Wydawnicza.

LA Rosa, Giuseppe (1996): La Sicilia come mito in Goethe e nei viaggiatori tedeschi. Palermo: Promopress.

LEED, ERIC J. (1992): La mente del viaggiatore. Bologna: Il Mulino.

ŁYSIAK, Waldemar (1974): Wyspy zaczarowane [Isole incantate]. Warszawa: Wyd. PAX.

MAGRIS, Claudio (1990): Introduzione. L'altrove narrato. Forme del viaggio in letteratura. Novara: Ist. Geografico De Agostini.

Olivieri, Mario (1997): Viaggi. Persistenze e variazioni nell'idea di viaggio. Perugia: Ed. Guerra.

OLKIEWICZ, Joanna (1975): Sycylia w kalejdoskopie historii i legendy [La Sicilia nel caleidoscopio della storia e della leggenda]. Warszawa: Ludowa Spółdzielnia Wydawnicza. 